\title{
Análisis Numérico de la Convección Mixta en un Conducto Horizontal de Sección Rectangular Tridimensional que Encierra un Escalón
}

\author{
Juan G. Barbosa-Saldaña*, José A. Jiménez-Bernal, Claudia del C. Gutiérrez-Torres y \\ Luis A. Moreno-Pacheco \\ Instituto Politécnico Nacional, Escuela Superior de Ingeniería Mecánica y Eléctrica, EPI/LABINTHAP, \\ Unidad Profesional Adolfo López Mateos, Av. Instituto Politécnico Nacional s/n, Edif. 5, $3^{\text {er }}$ Piso, \\ Col. Lindavista, 07738 México D.F.-México (e-mail: jbarbosas@ipn.mx, jgabriel_bs@yahoo.com.mx) \\ *autor a quien debe ser dirigida la correspondencia
}

\begin{abstract}
Resumen
Se realiza un estudio sobre la convección mixta para flujo laminar sobre un conducto horizontal que encierra un escalón. Las ecuaciones de momento y energía se discretizan usando la técnica de los volúmenes finitos. Se utiliza el algoritmo SIMPLE para enlazar los campos de velocidad y presión en el dominio computacional. La influencia de las fuerzas de flotación $(R i=3)$ sobre los campos de velocidad y de temperaturas, se simulan para un flujo de aire y un número de Reynolds constante $(\mathrm{Re}=200)$ y los resultados se comparan con los de flujo a convección forzada $(\mathrm{Ri}=0)$. Los resultados mostraron que las zonas de recirculación adyacentes al escalón, se ven reducidas cuando se consideran las fuerzas de flotación. Se concluye que el flujo es altamente tridimensional.
\end{abstract}

\section{Numerical Analysis for a Mixed Convective Flow Over a Tri-Dimensional Horizontal Backward-Facing Step}

\begin{abstract}
Laminar mixed convection over a backward-facing step is studied and presented in this work. A finite volume discretization technique is used to solve the momentum and energy equations. The SIMPLE algorithm is used to link the pressure distribution and velocity field inside the computational domain. The buoyancy forces affecting the velocity and temperature distributions are simulated for constant air flow and constant Reynolds number $(\mathrm{Re}=200)$ for a mixed convective flow $(\mathrm{Ri}=3)$ and the results are compared with those of pure force convective flow $(\mathrm{Ri}=0)$. The results indicate that the re-circulation zones at the vicinity of the back step are reduced if the buoyancy effects are considered. It is concluded that the flow is highly tridimensional.
\end{abstract}

Keywords: numerical simulation, mixed convection, forced convection, SIMPLE algorithm 


\section{INTRODUCCIÓN}

La separación y readherencia del flujo es un fenómeno que se encuentra en diversos equipos industriales, como es el caso de los intercambiadores de calor, enfriamiento de reactores nucleares, enfriamiento de alabes de turbinas, flujo en cámaras de combustión, flujo en difusores y válvulas. En algunas otras aplicaciones industriales, la separación es inducida para promover mejores condiciones de transferencia de calor y de masa, como es el caso de intercambiadores de calor compactos, cámaras de mezclado, combustores, etc. (Iwai et al., 2000a; Iwai et al., 2000b). Aún cuando muchos estudios han sido dedicados a entender y obtener un conocimiento más profundo de los aspectos hidrodinámicos y térmicos, vinculados con la separación y readherencia del flujo, todavía no se tiene una depuración de tales conceptos.

En la última década, gracias al desarrollo en la tecnología computacional, se han realizado una cantidad importante de estudios numéricos que analizan la dinámica de flujo y transferencia de calor en el fenómeno de la separación y readherencia del flujo. Particularmente, el punto que ha llamado más la atención de los investigadores es el fenómeno de flujo sobre un escalón. La razón de lo anterior es por que aún cuando la geometría es bastante sencilla, el flujo presenta fenómenos de separación y readherencia, sumado a características altamente tridimensionales. Incluso, es por esta razón que, el flujo sobre un escalón ha sido seleccionado como problema tipo para verificar y validar códigos y procedimientos numéricos (Blackwell y Pepper, 1992).

El estudio numérico y experimental sobre esta geometría ha sido un tema bastamente estudiado en la literatura, tanto en sus condiciones hidrodinámicas como el problema de convección forzada. Sin embargo, el problema de la convección mixta es un tema que debido a su complejidad y a la alta demanda de recursos computacionales, básicamente no ha sido estudiado. Algunos investigadores (Nie y Armaly, 2002a; Nie y Armaly, 2002b; Li y Armaly, 2000), han considerado el efecto de la convección mixta, pero sus trabajos se han centrado en conductos verticales, en los que las fuerzas de flotación y el flujo principal tienen la misma línea de acción. Por otra parte, Iwai et al. (2000a), presentan un estudio de la convección mixta en conductos con un escalón incluyendo los efectos de modificar la orientación del conducto. Sin embargo, estos autores en sus estudios reportan resultados para un régimen de Richardson modificado del orden de 0,03. Este rango es asociado con fuerzas de flotación extremadamente débiles, que no afectan considerablemente el campo de velocidad y, por lo tanto el fenómeno puede clasificarse como un problema de convección forzada.

Abu-Mulaweh (2003) presenta una recopilación de los trabajos numéricos y experimentales que se han desarrollado hasta la actualidad, y que tienen que ver con el flujo en conductos con un escalón. En su publicación, el autor no hace referencia a algún trabajo que presente resultados de la convección mixta en tres dimensiones dentro de un conducto horizontal con un escalón.

En este trabajo, se presentan los resultados numéricos obtenidos para la convección mixta en un conducto horizontal que tiene un escalón. Los resultados se presentan para un $\mathrm{Ri}=3$ y se comparan con los de la convección forzada para $\mathrm{Ri}=0$.

\section{MODELO MATEMÁTICO Y NUMÉRICO}

La geometría considerada en este estudio se presenta en la figura 1. La relación de aspecto (AR) y la relación de expansión (ER) del conducto en relación con la altura del escalón (s) son cuatro y dos respectivamente. La longitud total del conducto $(L)$ es igual a cincuenta y dos veces la altura del escalón, mientras que la longitud del escalón (l) en la dirección de la corriente es igual a dos veces la altura del mismo.

El fenómeno a estudiar es considerado como flujo estable y la aproximación de Boussinesq es utilizada para considerar los cambios de densidad del flujo debido a la convección mixta. Basados en tales simplificaciones, las ecuaciones de conservación de masa, de momento y de energía expresadas en forma tensorial se reducen a las siguientes formas, considerando que las propiedades físicas del aire permanecen constantes y son evaluadas a la temperatura promedio de entrada $\mathrm{T}_{0}$. 


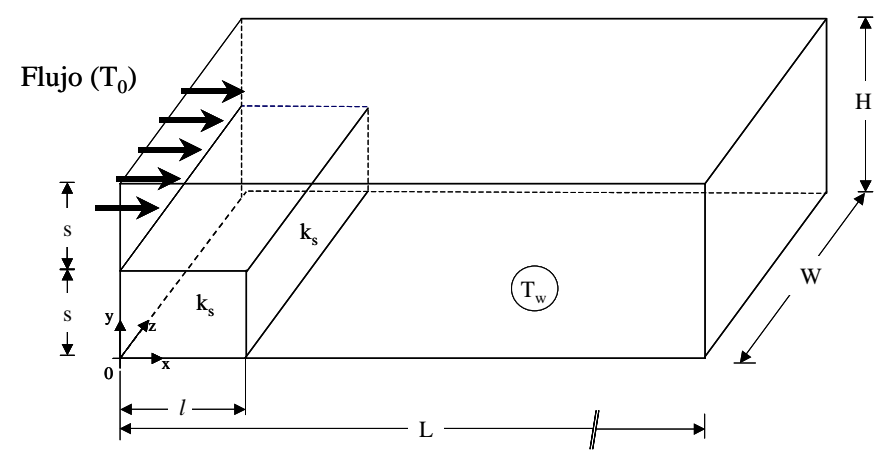

Fig. 1: Diagrama esquemático del conducto con el escalón en tres dimensiones

Ecuación de continuidad:

$\frac{\partial\left(\rho u_{i}\right)}{\partial x_{i}}=0$

Ecuación de momento:

$\frac{\partial\left(u_{j} u_{i}\right)}{\partial x_{i}}=-\frac{\partial p}{\partial x_{i}}+\frac{\partial}{\partial x_{j}}\left(\mu \frac{\partial u_{i}}{\partial x_{j}}+\mu \frac{\partial u_{j}}{\partial x_{i}}\right)+\rho_{\infty} g_{i} \beta\left(T-T_{\infty}\right)$

Ecuación de la energía:

$$
\frac{\partial}{\partial x_{i}}\left(\rho C p u_{i} T\right)=\frac{\partial}{\partial x_{i}}\left(k \frac{\partial T}{\partial x_{i}}\right)
$$

A la entrada del conducto, se considera un flujo de aire completamente desarrollado (Shah y London, 1978) e isotérmico $\left(T_{0}\right)$. La condición de no deslizamiento es considerada para todas las paredes del conducto, mientras que la pared inferior está sometida en su totalidad $(0 \leq x \leq L ; 0 \leq z \leq W)$ a una temperatura constante $\left(T_{w}\right)$, de tal forma que $T_{w}>T_{0}$, entre tanto que el resto de las paredes son consideradas como adiabáticas. La velocidad del flujo a la entrada del conducto es seleccionada de tal manera que el número de Reynolds tiene un valor de $\mathrm{Re}=200$ y se considera que el flujo es un flujo completamente desarrollado y tridimensional. Los efectos de las fuerzas de flotación en el campo de velocidades y la distribución de temperaturas son estudiadas para un número de Richardson $(\mathrm{Ri}=3)$ y los resultados se compararon con los de una convección forzada $(\mathrm{Ri}=0)$.

La técnica de los volúmenes finitos se implementó para discretizar la ecuación de momento y energía dentro del dominio computacional. El algoritmo SIMPLE (Patankar, 1980) se utilizó para unir la distribución de presiones y el campo de velocidades en las ecuaciones de momento. La solución a la ecuación de difusión-convección en una dimensión en la interfase de los volúmenes de control fue realizada por medio del esquema de la ley de potencias (Patankar, 1980). Una malla dislocada y no uniforme fue propuesta para resolver el problema dentro del dominio computacional. La malla es muy fina cerca de las paredes sólidas y en la vecindad del escalón, y va aumentando en forma progresiva en cada coordenada. La implementación del algoritmo de Thomas, línea-por-línea, en cada plano se utilizó para obtener la solución del campo de velocidades, presión y temperatura en el dominio computacional. La convergencia se declara cuando los residuos normalizados para los componentes de la velocidad y presión son menores a $10^{-8}$ y para la temperatura menor a $10^{-6}$.

El estudio de independencia de malla fue realizado para las condiciones mas severas $(\mathrm{R} i=3)$ y para varias densidades de nodos. En este caso, el punto de readherencia del flujo en el plano central en la dirección z, así como el número de Nusselt promedio a la salida del conducto, fueron los parámetros a monitorear para declarar independencia de malla. Los resultados indicaron que una malla de 100:40:40 (x:y:z) es suficiente para declarar independencia de malla (Barbosa et al., 2004). 
Como se comentó, el problema del flujo mixto convectivo en un conducto horizontal tridimensional con un escalón es un tema poco estudiado y no existen publicaciones que reporten datos de este problema. Tal carencia de datos hace de la validación del código numérico una tarea difícil de completar. Para cumplir con tan importante aspecto, se trató de validar el código con dos problemas muy semejantes al del flujo mixto convectivo en la geometría propuesta. El primer caso fue comparar los resultados del código con los resultados publicados para la convección forzada en un conducto horizontal con un escalón. Posteriormente se realizó la comparación de los resultados publicados para la convección mixta sobre un conducto horizontal y recto de sección transversal rectangular. En ambos casos la comparación entre los resultados del código desarrollado y los resultados publicados en la literatura mostraron una excelente concordancia, validando así el código numérico para esta investigación. Los resultados completos del procedimiento de validación pueden consultarse en publicaciones hechas por los autores previamente (Barbosa-Saldaña et al., 2004).

\section{RESULTADOS Y DISCUSIÓN}

De la literatura especializada, es bien sabido que el flujo sobre un escalón sufre una separación debido al cambio brusco en la geometría. Parte del flujo separado o desprendido, forma una zona de recirculación primaria que se encuentra adyacente al escalón, mientras que otra parte del flujo continua corriente abajo sin perturbación. La zona que delimita la recirculación primaria es la zona de readherencia del flujo. En esta zona, el flujo adyacente a la pared inferior del conducto inicia el desarrollo de una capa limite que corriente abajo se unirá o reagrupará con el flujo no perturbado y que dependiendo de las dimensiones del conducto, a la salida se tendrá un flujo completamente desarrollado. Sin embargo, el proceso del flujo antes descrito cambia su patrón completamente si se considera la presencia de las fuerzas de flotación. Este último escenario es el que corresponde a la convección mixta y es el tema principal en este trabajo. Para tal efecto, parámetros como la línea $x_{u}$, el número de Nusselt promedio, y algunos perfiles de velocidad en planos específicos son utilizados para analizar los efectos de la convección mixta en el flujo dentro de un conducto tridimensional con un escalón, como se expone a continuación.

Una definición importante para el caso de flujo sobre un escalón en dos dimensiones, es el punto de readherencia o punto donde el flujo se reúne después de la separación. Este punto tiene como particularidad que el esfuerzo cortante en la pared es igual a cero y comúnmente se utiliza para definir o delimitar la zona de recirculación primaria. Sin embargo, para el caso tri-dimensional dicho punto no puede utilizarse para delimitar la zona de recirculación adjunta al escalón. En este caso, se recurre a un concepto similar que es la línea $x_{u}$. Esta línea sirve para delimitar la zona de recirculación primaria a lo ancho del canal y numéricamente se obtiene por los puntos en los cuales la componente en la dirección del flujo cambia de un valor negativo a un valor positivo. La gráfica de la línea $x_{u}$ para el caso de $R i=3$ y $R i=0$ se muestra en la figura 2.

El comportamiento de la línea $x_{u}$ es simétrico respecto al plano central en la dirección $z \quad(z / W=0,5)$ debido a la geometría del conducto, y en ambos casos la forma que se asemeja para dicha distribución es la de una "W". Es en la zona adyacente a las paredes del conducto donde se encuentran los puntos más retardados de readherencia del flujo. Está situación se atribuye a que en esta zona el momento es menor que en la zona central y por lo tanto es más difícil para el flujo vencer los gradientes de presión adversos debidos a la separación del flujo y por lo tanto el cambio del componente de la velocidad principal de negativo a positivo necesita una mayor longitud para ocurrir. Es evidente en la figura 2 que la zona de recirculación primaria adyacente al escalón se ve reducida si el Ri es incrementado.

De acuerdo con la literatura el flujo mixto convectivo en conductos presenta dos diferentes estructuras que dependen de la naturaleza de la convección mixta y por lo tanto del parámetro de Richardson (Luijkx et al., 1991). La presencia de roles longitudinales (eje paralelo al eje x) está asociada a números elevados de Grashoff. Por lo tanto, la presencia de estas estructuras de flujo provoca que la zona primaria de recirculación se vea reducida. Por otra parte, la convección mixta implica la acción de fuerzas de flotación que están íntimamente ligadas al componente vertical de la velocidad y que contribuyen de igual manera a reducir la zona de recirculación primaria. 
La distribución a lo largo del conducto para el número de Nusselt promedio se presenta en la figura 3. A la entrada del conducto, las distribuciones para convección mixta $(\mathrm{Ri}=3)$ y convección forzada $(\mathrm{R} i=0)$ presentan valores elevados que exponencialmente decrecen. Ambas distribuciones presentan un comportamiento similar a lo largo del conducto y se aprecia que a mayor número de Richardson, mayores son los valores para el número de Nusselt. En la vecindad del escalón $(x / s=2)$ se presentan los valores mínimos para esta distribución e incluso se puede apreciar una discontinuidad. Este efecto debe de interpretarse como el efecto de la separación del flujo debido al cambio brusco en la geometría del conducto. Después de presentar el mínimo valor, ambas distribuciones tienen un crecimiento repentino que se debe a los efectos de la zona primaria de recirculación. Es en esta zona donde se dan las razones de transferencia de calor más elevadas. Posterior a alcanzar su valor máximo, ambas distribuciones decrecen exponencialmente hacia la salida del conducto.

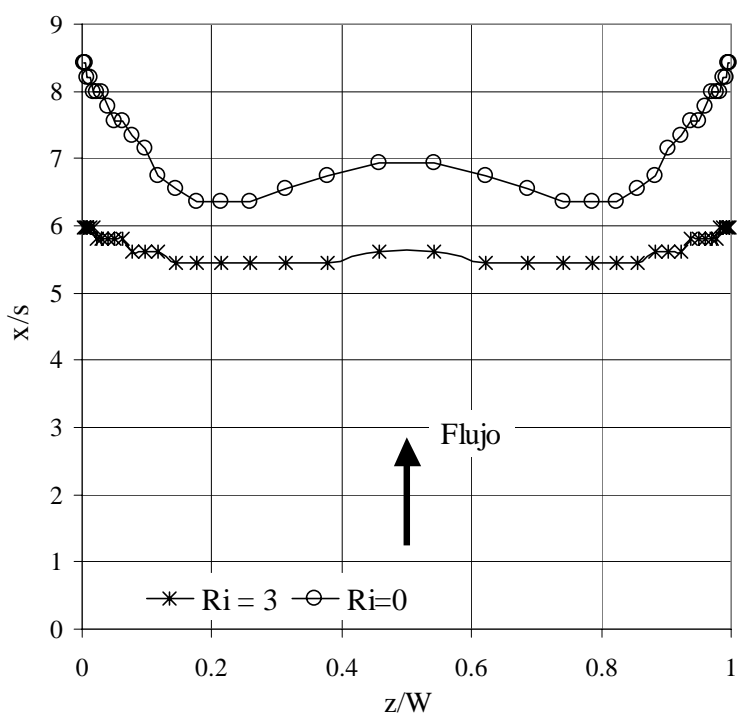

Fig. 2: Distribución de la línea $x_{u}$ para el flujo tridimensional sobre un escalón

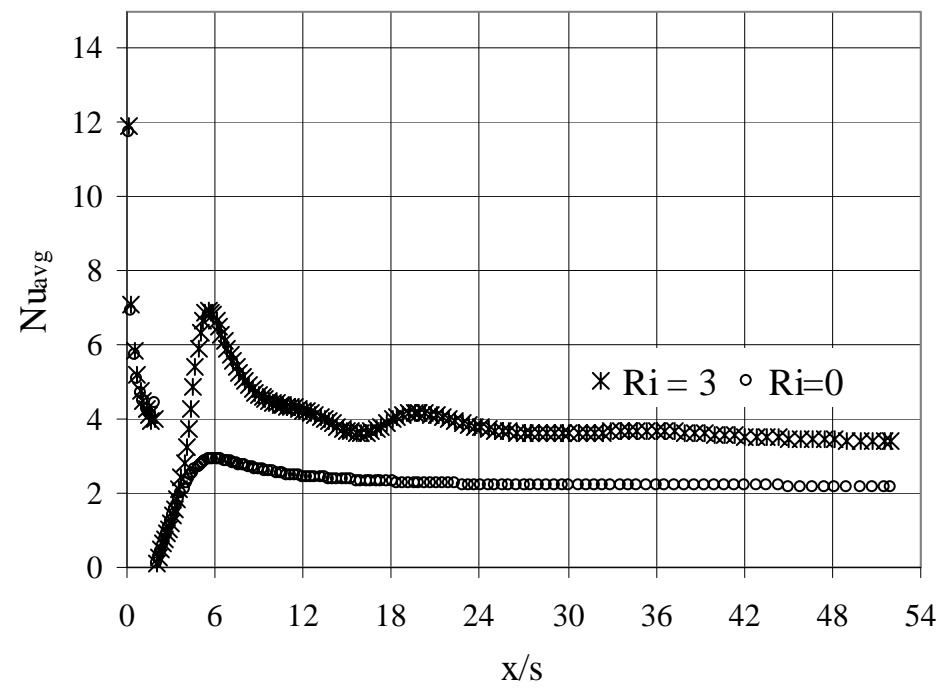

Fig. 3. Distribución del número de Nusselt promedio a lo largo del canal con escalón

La distribución de velocidades en el plano central en la dirección z ( $\mathrm{z} / \mathrm{W}=0,5)$, para la componente $u$ de la velocidad a diferentes posiciones en la dirección $x$, se muestra en la figura 4 . Como se mencionó, a la entrada el flujo se considera hidrodinámicamente como un flujo completamente desarrollado $(x / s=0)$. En la vecindad del escalón $(x / s=2)$, la componente u de la velocidad tiene pequeñas desviaciones de los valores de flujo completamente desarrollado en la parte superior del conducto $(y / s>0,5)$. Por otra parte, en la parte inferior del conducto $(y / s<0,5)$ se observa que la componente $u$ presenta valores negativos que se asocian a la zona de recirculación adyacente al 
escalón. Este último efecto se ve más pronunciado para el caso de $\mathrm{Ri}=3$. Para el valor de $\mathrm{x} / \mathrm{s}=4,7 \mathrm{la}$ recirculación del flujo es evidente. A medida que el número de Richardson se incrementa, la zona de recirculación en la dirección vertical se ve reducida, este fenómeno se asocia a los efectos de flotación.

A la distancia de $x / s=26$ (mitad del conducto), se observa que para $R i=0$ la componente $u$ de la velocidad tiende a formar el perfil de flujo completamente desarrollado, incluso se observa que para este caso el conducto, es lo suficientemente largo para acomodar un flujo completamente desarrollado a la salida del mismo. Sin embargo, para $\mathrm{Ri}=3$ se observa que el flujo a la salida tiene una forma inconstante y que el valor de la velocidad máxima se encuentra desplazado hacia la parte inferior del conducto. La razón de este comportamiento será explicada después de considerar la figura 5, la cual muestra los vectores de velocidad y la distribución de temperaturas en diferentes planos $\mathrm{x}$.

Las estructuras de flujo mostradas en la figura $5 \mathrm{a}$, indican que para $\mathrm{Ri}=3$ se forman un par de roles longitudinales, cuyo eje axial es paralelo al eje $x$. Es evidente en esta figura que el flujo sube por las paredes laterales y se desplaza en la dirección opuesta en la parte central del conducto $(z=0,02)$. Esta es la razón por la cual el componente máximo de la velocidad a la salida del conducto en la figura $4 a$ se localiza en la parte inferior del conducto. Para el caso de Ri=0 (figura $5 b$ ), las estructuras de flujo no muestran la formación de los roles convectivos. Sin embargo, se puede apreciar que los vectores de velocidad son paralelos al eje $x$, por lo que el flujo es completamente desarrollado como se había mencionado.

La distribución de temperaturas en la figura $5 \mathrm{a}$, muestra que se encuentra una zona de alta temperatura estratificada en la parte superior del conducto. Este resultado es esperado, ya que por efectos de la convección mixta, el flujo con menor densidad y mayor temperatura debe de colocarse en la parte superior. De la misma forma, se observa que si se considera un plano y=constante, la distribución de temperaturas indica que las temperaturas más elevadas se encuentran en la vecindad de las paredes laterales. Este comportamiento se da debido a que el flujo asciende por las paredes laterales y desciende por la parte central del conducto.

La distribución de temperaturas para convección forzada ( $\mathrm{Ri}=0$ ) (figura $5 \mathrm{~b}$ ) presenta un comportamiento completamente diferente al de convección mixta $(\mathrm{Ri}=3)$. En el caso de convección forzada, la zona de mayor temperatura se localiza adyacente a la pared inferior del conducto porque es la zona de calentamiento según las condiciones del problema. Como se puede deducir de la figura $5 a$, las estructuras de flujo para convección mixta son extremadamente complicadas. La formación de los dos roles longitudinales es consecuencia de las fuerzas de flotación. A continuación se hace un descripción de la formación de estas estructuras a lo largo de la dirección principal del flujo para la convección mixta y se utiliza la figura 6 para presentar los vectores de velocidad y las diferentes estructuras de flujo en diferentes planos.
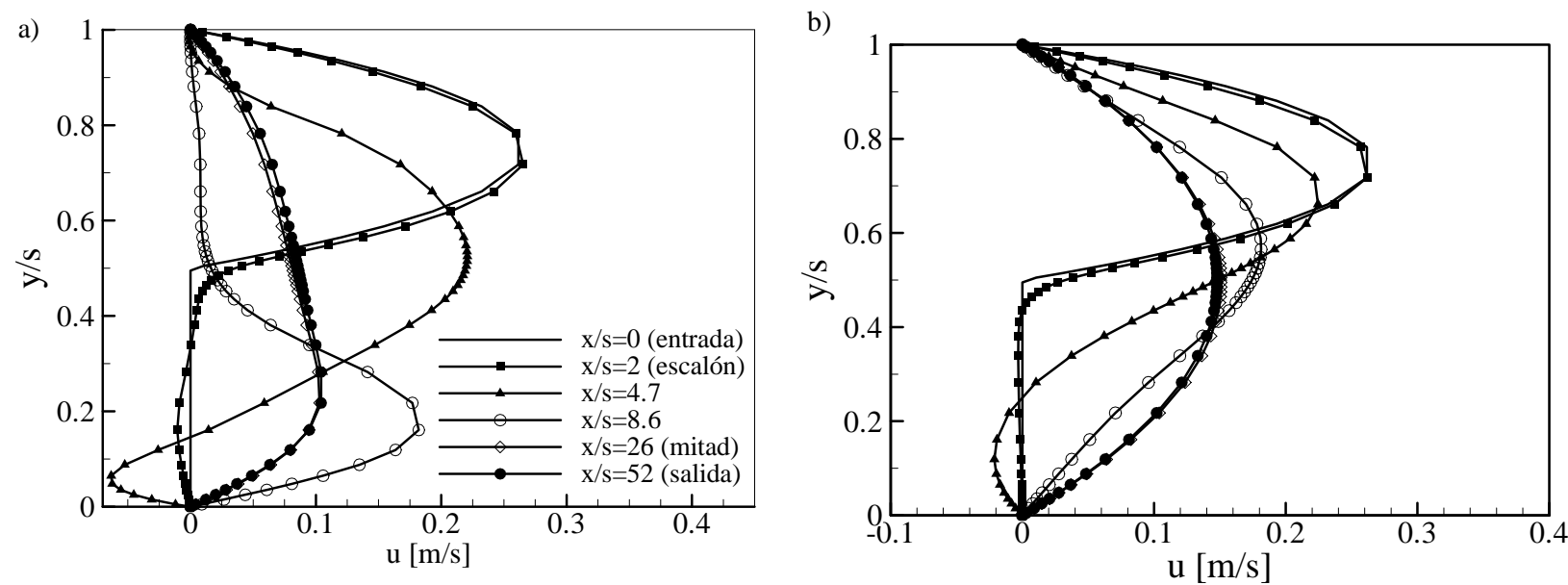

Fig. 4: Perfiles de la componente $u$ de la velocidad para el plano $z / W=0.5$ a diferentes posiciones $x$ a) $\mathrm{Ri}=3$ b) $\mathrm{Ri}=0$ 
a)
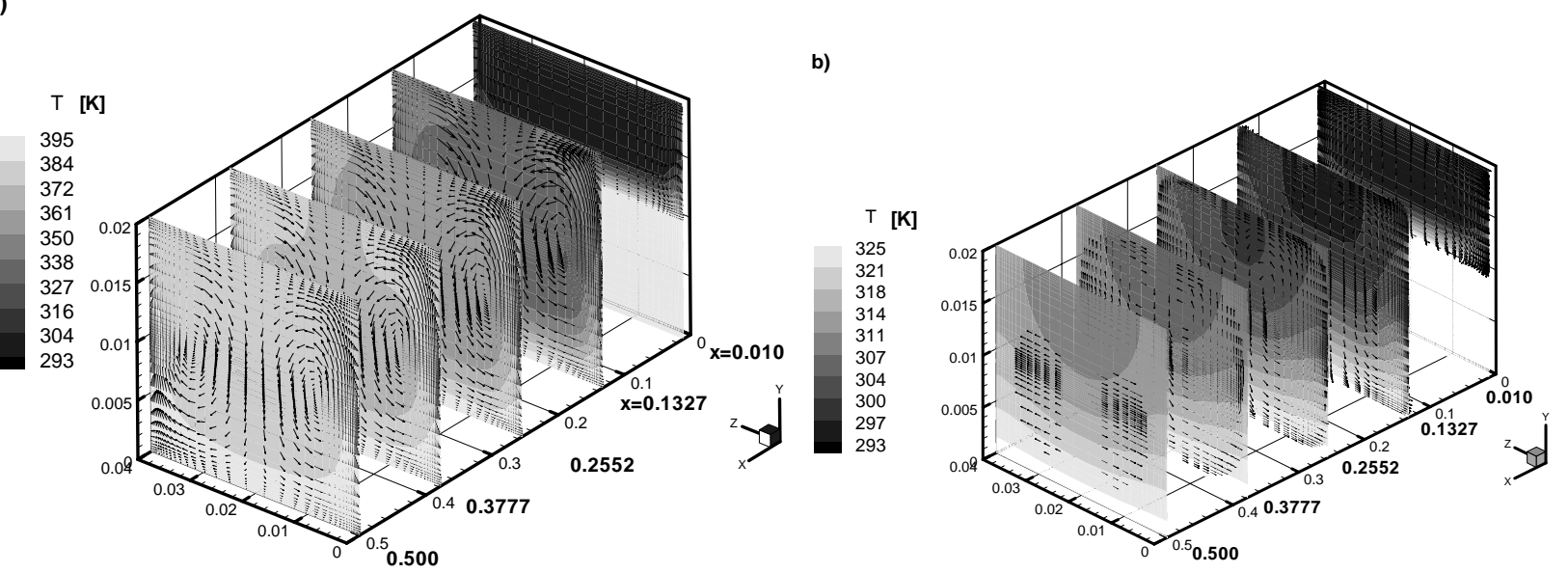

Fig. 5: Contornos de temperatura y vectores de velocidad a diferentes planos $\times$ a) $R i=3, b) R i=0$
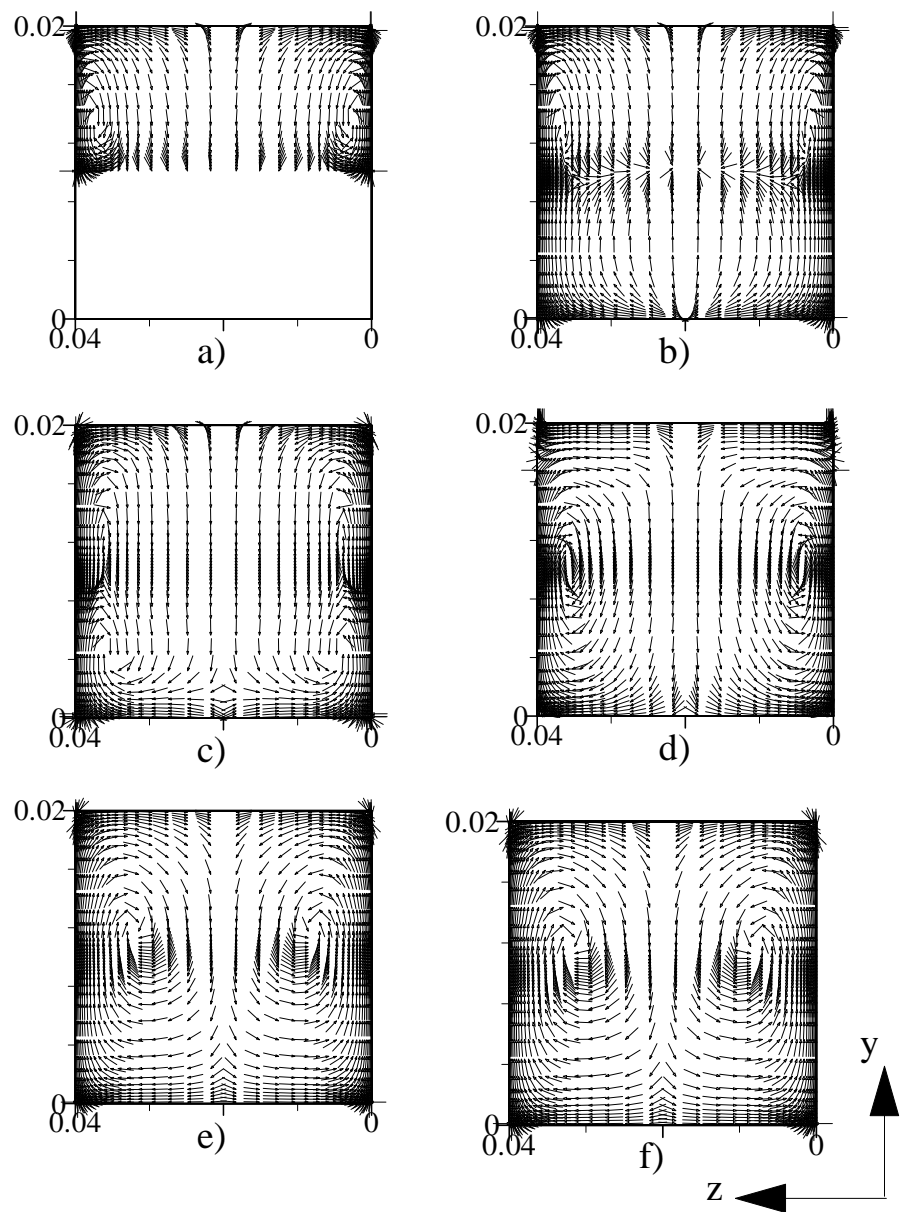

Fig. 6. Vectores de velocidad para $\mathrm{Ri}=3 \mathrm{a}) \mathrm{x} / \mathrm{s}=1,8 \mathrm{~b}$ ) $\mathrm{x} / \mathrm{s}=2$ (escalón) $\mathrm{c}$ ) $\mathrm{x} / \mathrm{s}=3,2 \mathrm{~d}$ ) $\mathrm{x} / \mathrm{s}=4,7$ e) $\mathrm{x} / \mathrm{s}=10$ f) $x / s=52$ (salida)

En la figura $6 a$, se muestra un plano constante $x / s=1,8$ que es una región previa al escalón. El flujo presenta la formación de pequeños vórtices en las esquinas del conducto debido a la condición de no deslizamiento impuesta a todas las paredes del conducto. También se observa que los vectores de velocidad tienden a dirigirse hacia la parte baja del conducto, razón que se entiende debido al cambio en la geometría que se esta estudiando. En la medida que el flujo pasa a una posición posterior al escalón ( $\mathrm{x} / \mathrm{s}>2$ ), se observa que el flujo se desplaza hacia las paredes laterales del 
conducto (figura 6b). También se puede apreciar que la dirección de los vectores de velocidad en la parte superior del conducto tiende a desplazarse hacia abajo, mientras que en la parte inferior del conducto, los vectores de velocidad indican que la tendencia es que el flujo se desplace en la dirección y positiva (hacia arriba). Como consecuencia, en la región central del conducto en la dirección vertical $(y=0,01)$ las estructuras de flujo muestran una zona donde dos corrientes se están encontrando, la que baja de la parte superior por el cambio en la geometría y la que sube porque el flujo rebota en la pared inferior del canal.

La figura 6c muestra que en esta zona del conducto la tendencia del flujo es la de desplazarse hacia las paredes laterales y también el efecto de que el flujo se mueve de la parte inferior a la superior y viceversa. Esta posición del conducto $(x / s=3,2)$ se encuentra en la zona de la recirculación primaria, zona en la cual el flujo es altamente tridimensional, sobre todo en la parte inferior del conducto. La siguiente posición a considerar es para $(x / s=4,7)$. En esta zona ya se identifican plenamente los roles convectivos atribuibles a la convección mixta en el conducto (figura 6d). Aunado a esto, se observa que el flujo asciende por las paredes laterales mientras que se desplaza hacia la parte inferior por la zona central $(z=0,02)$ del conducto.

Las figuras $6 \mathrm{e}$ y $6 \mathrm{f}$ muestran las estructuras de flujo a una distancia $\mathrm{x} / \mathrm{s}=10$ y a la salida del conducto, respectivamente. En estas figuras se puede apreciar con claridad la formación de los roles convectivos longitudinales. Si se comparan la figuras 6 e y $6 \mathrm{~d}$, se observa que para la primera, el vórtice del rol se ha desplazado hacia la zona central del conducto en la dirección transversal. Este desplazamiento indica que los roles convectivos presentan una estructura tipo espiral cuyo eje axial no es paralelo al eje $\mathrm{x}$. La razón por la cual se presenta este particular fenómeno se atribuye a la formación de zonas estratificadas de temperatura a lo largo de la coordenada transversal del conducto (coordenada z) y a lo largo de la coordenada vertical del mismo (coordenada y), como se observa en las figuras $5 a$ y $5 b$.

\section{CONCLUSIONES}

El fenómeno de la convección mixta a través de un conducto horizontal con un escalón fue simulado numéricamente aplicando la técnica de los volúmenes finitos.

Las fuerzas de flotación fueron consideradas para un número de Richardson $\mathrm{R} i=3$ y los resultados se compararon con los de convección forzada. Los resultados indicaron que las fuerzas de flotación tienen una significativa influencia en el campo de velocidades y en la distribución de temperaturas cuando se comparan los regímenes de convección mixta y convección forzada.

El punto donde se obtiene el máximo valor para el número de Nusselt aparece corriente arriba si el parámetro de Ri se incrementa. Así mismo, la zona de recirculación primaria adyacente al escalón se ve considerablemente reducida; no solamente en la dirección del flujo principal, sino también en la dirección vertical del conducto, cuando se considera los efectos de la convección mixta.

Las estructuras del flujo mixto indican la formación de roles longitudinales convectivos que se forman a lo largo del conducto, este fenómeno implica que el flujo es altamente tridimensional.

La futura investigación relacionada con este fenómeno permitirá dar un panorama más amplio y una explicación más profunda de las estructuras de flujo y de la distribución de temperaturas en esta geometría.

\section{NOMENCLATURA}

AR Relación de aspecto, $A R=W / s$

Cp Calor específico [J/kgK]

ER Relación de expansión, ER=H/s

g Aceleración de la gravedad $\left[\mathrm{m} / \mathrm{s}^{2}\right]$ 
Gr Número de Grashof $\left[g \beta\left(T_{w}-T_{0}\right) s^{3} / v^{2}\right]$

$\mathrm{H} \quad$ Altura del conducto a la salida [m]

$\mathrm{k} \quad$ Conductividad térmica $[\mathrm{W} / \mathrm{mK}]$

$\mathrm{L} \quad$ Longitud del conducto, $\mathrm{L}=52 \mathrm{~s}[\mathrm{~m}]$

$l \quad$ Longitud del escalón, $l=2 \mathrm{~s}[\mathrm{~m}]$

$\mathrm{Nu} \quad$ Número de Nusselt, $\left.[2 s(d T / d y))_{y=0} /\left(T_{w}-\bar{T}\right)\right]$

p Presión [Pa]

Re Número de Reynolds $\left[2 \rho U_{0} \mathrm{~s} / \mu\right]$

Ri Número de Richardson; [ $\mathrm{Gr} / \mathrm{Re}^{2}$ ]

$\mathrm{s} \quad$ Altura del escalón [0.01 m]

$\mathrm{T} \quad$ Temperatura [K]

W Ancho del conducto [m]

$\mathrm{x}, \mathrm{y}, \mathrm{z}$ Direcciones coordenadas

\author{
Subíndices \\ 0 entrada \\ w pared \\ $\infty \quad$ valor de corriente libre
}

Letras griegas

$\beta \quad$ Coeficiente de expansión térmica $\left[\mathrm{K}^{-1}\right]$

$\mu \quad$ Viscosidad dinámica $[\mathrm{kg} / \mathrm{ms}]$

$v \quad$ Viscosidad cinemática $\left[\mathrm{m}^{2} / \mathrm{s}\right]$

$\rho \quad$ Densidad $\left[\mathrm{kg} / \mathrm{m}^{3}\right]$

\title{
REFERENCIAS
}

Abu-Mulaweh, H.I.; A review of research on laminar mixed convection flow over a backward-and forward-facing steps, Int. Journal of Thermal Sciences, 42, 897-909 (2003).

Barbosa-Saldaña, J.G., N.K. Anand y V. Sarin; Numerical Simulation of Mixed Convective Flow over a Three-Dimensional Horizontal Backward-Facing Step, Proceedings of ASME-HT-FED04, Charlotte, North Carolina, USA (2004).

Blackwell, B.F. y D.W. Pepper; Benchmark problems for heat transfer codes, Proceedings of ASMEHTD-222, NY., USA (1992).

Iwai, H., K. Nakabe, K. Suzuki y K. Matsubara; Flow and heat transfer characteristics of backwardfacing step laminar flow in a rectangular duct, Int. J. Heat and Mass Transfer, 43, 457-471 (2000a).

Iwai, H., K. Nakabe, K. Suzuki y K. Matsubara; The effects of duct inclination angle on laminar mixed convective flows over a backward-facing step, Int. J. Heat and Mass Transfer, 43, 473-485 (2000b).

Li, A. y B.F Armaly; Mixed convection adjacent to a 3-D backward facing step, Proceedings of ASMEHTD, 2, 51-58 (2000).

Luijkx, J.M., J.K. Platten y J.C.L. Legros; On the existence of thermoconvective rolls, transverse to a superimposed mean Poiseuille flow, Int. J. Heat and Mass Transfer, 24, 1287-1291 (1991). 
Nie, J.H. y B.F. Armaly; Buoyancy effects on three-dimensional convective flow adjacent to backwardfacing step, AIAA J. Thermophysics and Heat Transfer, 17, 122-126, (2002a).

Nie, J.H. y B.F. Armaly; Three-Dimensional Convective Flow Adjacent to Backward-Facing StepEffects of step height, Int. J. Heat and Mass Transfer, 45, 2431-2438 (2002b).

Patankar, S.V.; Numerical heat transfer and fluid flow, Taylor \& Francis Inc., Philadelphia, USA (1980).

Shah, R.K. y A.L. London; Laminar flow forced convection in ducts, Academic Press, New York, USA (1978). 